\title{
Nonperturbative Flow Equations from Running Expectation Values
}

\author{
F. G. Scholtz, B. H. Bartlett, and H. B. Geyer \\ Institute of Theoretical Physics, University of Stellenbosch, Stellenbosch 7600, South Africa
}

(Received 22 January 2003; published 21 August 2003)

\begin{abstract}
We show that Wegner's flow equations, as recently discussed in the Lipkin model, can be solved selfconsistently. This leads to a nonlinear differential equation which fully determines the order parameter as a function of the dimensionless coupling constant, even across the phase transition. Since we consider an expansion in the fluctuations, rather than the conventional expansion in the coupling constant, convergence to the exact results is found in both phases when taking the thermodynamic limit.
\end{abstract}

DOI: 10.1103/PhysRevLett.91.080602

General nonperturbative techniques to solve interacting quantum systems are notoriously difficult to construct, and this is probably the greatest obstacle to a thorough understanding of such systems and, in particular, their phase structure. One technique that, at least in principle, should shed light on these aspects is the renormalization group and associated flow equations [1]. It is probably for this reason that considerable interest was generated in the flow equations recently proposed by Wegner and independently by Glazek and Wilson [2] as well as its demonstrated close relation to the renormalization group [3]. Further applications of the flow equations, based on successive infinitesimal unitary transformations, to diverse quantum mechanical problems followed, including the treatment of the electronphonon coupling [2], the spin-boson Hamiltonian [4], the Hubbard model [5], the sine-Gordon model [6], and the Foldy-Wouthuysen transformation [7]. The Lipkin model was particularly prominent among applications [8-10], probably because it is a many-body model that can be solved numerically quite easily, but which exhibits a nontrivial quantum phase transition [11] and could therefore shed light on the practical implementation of these flow equations in a simple, and yet nontrivial setting.

Generally the Hamiltonian does not preserve its form under the flow, and additional operators, not present in the original Hamiltonian, are generated. This is generally the case under a renormalization group transformation [1], and certainly also the case in Wegner's flow equations [2]. The generic situation yields an infinite set of nonlinear coupled differential equations for the coupling constants, and some form of truncation is required [12]. Here we propose a novel truncation procedure. This entails the expansion of many-body operators, generated during the flow, in terms of the fluctuations of their one-body constituents around their ground-state expectation values. In this way the form of the original Hamiltonian, e.g., one plus two-body interactions, can be maintained. The novel aspect here, which goes well beyond the simplest implementation of Ref. [8], is that the unknown ground-state expectation values are determined self-consistently as they evolve under the flow. This yields as a by-product a
PACS numbers: 05.10.Cc, 03.65.-w, 11.10.Hi, 64.60.-i

set of equations which determines these expectation values as functions of the dimensionless coupling constants. This is in contrast with [8] where the flow of the expectation values is not taken into account. One expects that the effective Hamiltonian obtained in this way will describe the low-energy dynamics well. Instead of elaborating a general formulation of the above central idea, we rather demonstrate it in the Lipkin model. This standard and widely used test model for many-body techniques allows a transparent discussion, and yet it is general enough to illustrate the main points of the construction.

In the Lipkin model [11] $N$ fermions distribute themselves on two $\Omega$-fold degenerate levels which are separated by an energy $\xi_{0}$. The interaction $V_{0}$ introduces a scattering of pairs between the two shells. Labeling the two levels by $\sigma= \pm 1$, the Hamiltonian reads

$$
H_{0}=\xi_{0} \sum_{\sigma, p} \sigma a_{p, \sigma}^{\dagger} a_{p, \sigma}+\frac{V_{0}}{2} \sum_{p p^{\prime}, \sigma} a_{p, \sigma}^{\dagger} a_{p^{\prime}, \sigma}^{\dagger} a_{p^{\prime},-\sigma} a_{p,-\sigma} .
$$

A spin representation for $H$ may be found by introducing the $s u(2)$ generators

$$
J_{z}=\frac{1}{2} \sum_{p, \sigma} \sigma a_{p, \sigma}^{\dagger} a_{p, \sigma}, \quad J_{ \pm}=\sum_{p} a_{p, \pm 1}^{\dagger} a_{p, \mp 1} .
$$

The Hamiltonian commutes with $J^{2}$ and acts in the irreducible representations of $s u(2)$, leading to a block diagonal structure of size $2 j+1$. The low-lying states occur in the multiplet $j=N / 2$ [11]. To remove a trivial scaling factor we divide by $\xi_{0}$ and define the dimensionless coupling constant $\beta_{0}=2 j V_{0} / \xi_{0}$; i.e., all results are expressed in units of $\xi_{0}$ :

$$
H_{0}\left(\beta_{0}\right)=J_{z}+\frac{\beta_{0}}{4 j}\left(J_{+}^{2}+J_{-}^{2}\right) .
$$

With no interaction $\left(\beta_{0}=0\right)$ the ground state is the product state $\left|\psi_{0}\right\rangle=\prod_{i=1}^{N} a_{i,-1}^{\dagger}|0\rangle$ which is written in the spin basis as $\left|\psi_{0}\right\rangle=|j=N / 2,-j\rangle$, and $\left\langle\psi_{0}\left|J_{z}\right| \psi_{0}\right\rangle=-j$. Turning the interaction on leads to particle-hole excitations across the gap (undeformed phase, $\beta_{0}<1$ ) and at the critical value $\beta_{0}=1$ the model shows a phase change 
to a ground state of condensate pairs (deformed phase, $\beta_{0}>1$ ). As usual the phase transition is sharp only in the thermodynamic limit $(N \rightarrow \infty)$, while it gets smoothed out by fluctuations at finite $N$. An order parameter that distinguishes between the two phases is $\Omega \equiv 1+\left\langle J_{z}\right\rangle / j$, where $\left\langle J_{z}\right\rangle$ is the expectation value of $J_{z}$ in the ground state. A detailed discussion of the features of the model can be found in Refs. [11,13].

In previous applications of the flow equations to the Lipkin model [8-10] a variety of procedures were followed to close the flow equations into a set of nonlinear differential equations for a few parameters. In all cases this worked well in the undeformed phase, but led to divergent results in the deformed phase. This is a manifestation of the fact that the two different phases (regions of coupling) flow to different fixed points, which cannot be accommodated by the approximations made. Here we present a self-consistent way of closing the flow equations which can accommodate the fixed points of both phases.

Applying Wegners's flow equations to (3) we have the differential equation

$$
\frac{d H(\ell)}{d \ell}=[\eta(\ell), H(\ell)], \quad H(0)=H_{0}\left(\beta_{0}\right)
$$

with an appropriate choice of the anti-Hermitian generator $\eta$. The original Hamiltonian $H_{0}$ is band diagonal, and we choose our generator as in [8],

$$
\eta(\ell)=\left[J_{z}, H(\ell)\right],
$$

since direct computation shows that this choice preserves the band diagonal structure. Furthermore, as is well known $[2,3,8]$, the fixed point Hamiltonian commutes with $J_{z}$ and is therefore diagonal in the eigenbasis of $J_{z}$, so that the eigenvalues can be read off directly.

To close the flow equations we generalize the approach of [8] and parametrize $H(\ell)$ as

$$
H(\ell)=\alpha(\ell) J_{z}+\beta(\ell) \frac{1}{4 j}\left(J_{+}^{2}+J_{-}^{2}\right)+\delta(\ell) j
$$

with initial conditions $\alpha(0)=1, \beta(0)=\beta_{0}, \delta(0)=0$. Here a $\delta$ term proportional to the identity has been included. Substitution into expressions (4) and (5) yields

$$
\begin{aligned}
\eta= & {\left[J_{z}, H(\ell)\right]=\frac{\beta}{2 j}\left(J_{+}^{2}-J_{-}^{2}\right), } \\
{\left[\left[J_{z}, H(\ell)\right], H(\ell)\right]=} & \beta^{2} \frac{2 j(j+1)-1}{j^{2}} J_{z}-\frac{2 \beta^{2}}{j^{2}} J_{z}^{3} \\
& -\frac{4 \alpha \beta}{4 j}\left(J_{+}^{2}+J_{-}^{2}\right)
\end{aligned}
$$

in which a term $\sim J_{z}^{3}$ has been generated. As in [8] we approximate the effect of this term on the low-lying states by linearizing around the ground-state expectation value and neglecting higher-order fluctuations, i.e., $J_{z}^{3} \mapsto 3\left\langle J_{z}\right\rangle^{2} J_{z}-2\left\langle J_{z}\right\rangle^{3}$, to give

080602-2

$$
\begin{aligned}
& \dot{\alpha}=-\beta^{2}\left(\frac{6\left\langle J_{z}\right\rangle^{2}-2 j(j+1)+1}{j^{2}}\right), \\
& \dot{\beta}=-4 \alpha \beta, \\
& \dot{\delta}=4 \beta^{2} \frac{\left\langle J_{z}\right\rangle^{3}}{j^{3}} .
\end{aligned}
$$

The difference between our approach and that of [8] is that in the latter $\left\langle J_{z}\right\rangle$ is evaluated with respect to the state $|j=N / 2,-j\rangle$ throughout the flow which gives $\left\langle J_{z}\right\rangle=-j$. Because of the different types of behavior of this expectation value in the two phases, this is not sufficient if one wants to describe the low-energy dynamics of both phases. Instead, one has to linearize around the ground-state expectation value $\left\langle J_{z}\right\rangle$ which will be different in the respective phases. Furthermore, one has to keep in mind that the ground state of the effective Hamiltonian changes (undergoes a unitary transformation) during the flow, so that one actually has to linearize around the ground-state expectation $\left\langle J_{z}\right\rangle(\ell)$ of $J_{z}$ at any given point in the flow. At first sight the latter is an unknown function, but we shall see shortly how this function can be determined in a self-consistent manner. It is also important to note that the constant term appearing in the linearized form of $J_{z}^{3}$ will generate a term proportional to the identity, which will not affect the gap $\Delta$ between the ground and first excited states, but which will affect the ground-state energy, and therefore it has to be included if one wants to compute the latter.

We now proceed to set up an equation for the unknown function $\left\langle J_{z}\right\rangle(\ell)$. We first note that this function can depend only on the parameters determining the ground state of (6). In particular, this implies that this function has no explicit $\ell$ dependency, but that its $\ell$ dependency is implicit through its dependence on $\alpha(\ell), \beta(\ell)$, and $\delta(\ell)$. Since a global rescaling of the Hamiltonian and the addition of a constant rescales and shifts the eigenvalues, while the eigenstates are unaffected, this function can depend only on the dimensionless coupling constant $x(\ell) \equiv$ $\beta(\ell) / \alpha(\ell)$. Denoting by $|G, \ell\rangle$ the ground state of the system at the point $\ell$ in the flow, this implies that we can write $\left\langle J_{z}\right\rangle(\ell) \equiv\left\langle G, \ell\left|J_{z}\right| G, \ell\right\rangle \equiv f[\beta(\ell) / \alpha(\ell)]$.

We recall a few basic facts about unitary flow equations [2]: the ground state $|G, \ell\rangle$ is related to the ground state at $\ell=0$ by a unitary transformation of which $\eta$ is the generator, i.e., $|G, \ell\rangle=U(\ell)|G, \ell=0\rangle$ and $\eta=\frac{d U}{d \ell} U^{\dagger}=$ $-U \frac{d U^{\dagger}}{d \ell}$. By construction the eigenvalues are invariants of the flow, and, in particular, the ground-state energy $E_{g}=$ $\langle G, \ell|H(\ell)| G, \ell\rangle=\langle G, \ell=0|H(0)| G, \ell=0\rangle$. Differentiating $f[\beta(\ell) / \alpha(\ell)]$ with respect to $\ell$ and using these facts together with the flow equations (8) we obtain

$$
\left[\frac{\beta^{3} F(\beta / \alpha)}{j^{2} \alpha^{2}}-4 \beta\right] f^{\prime}(\beta / \alpha)=-\left\langle G, \ell\left|\left[\eta, J_{z}\right]\right| G, \ell\right\rangle,
$$

where we have introduced the function $F(x)=6 f^{2}(x)-$ $2 j(j+1)+1$. Using the result for $\eta$ in (7) and the parametrization (6) this can be written as 


$$
\left[\frac{\beta^{3} F(\beta / \alpha)}{j^{2} \alpha^{2}}-4 \beta\right] f^{\prime}(\beta / \alpha)=4\left[E_{g}-\alpha f(\beta / \alpha)-j \delta\right] .
$$

Differentiating this result again with respect to $\ell$ and using the flow Eqs. (8) we obtain the final result, written in terms of the dimensionless coupling constant $x$,

$$
\begin{array}{r}
{\left[\frac{x^{2} F(x)}{j^{2}}-4\right]^{2} f^{\prime \prime}(x)+\left[\frac{2 x^{3} F^{2}(x)}{j^{4}}-\frac{8 x F(x)}{j^{2}}\right] f^{\prime}(x)+} \\
\frac{12 x^{4} F(x)}{j^{4}} f^{\prime 2}(x) f(x)-\frac{48 x^{2}}{j^{2}} f^{\prime 2}(x) f(x)- \\
\frac{4 F(x)}{j^{2}} f(x)+\frac{16}{j^{2}} f^{3}(x)=0 .
\end{array}
$$

This is a nonlinear differential equation that uniquely determines the function $f(x)$, and thus the order parameter $\Omega=1+f(x) / j$, as a function of the dimensionless coupling constant $x$, once boundary conditions have been specified. In principle, this equation therefore contains all information on the phase structure of the system. To determine the boundary conditions on $f(x)$ we note that at $x=0$ the Hamiltonian is $H=J_{z}$ so that $f(0)=$ $-j=-N / 2$. Furthermore from $f(-x)=f(x)$, one easily sees that $f(x)$ attains its minimum value at $x=0$. Thus we have the boundary conditions $f(0)=-j=$ $-N / 2, f^{\prime}(0)=0$.

In Fig. 1 we display the order parameter $\Omega$ as a function of $x$ for $j=N / 2=25$. We also show the exact result as obtained from a diagonalization of the Hamiltonian. We note excellent agreement, even in the transitional regions where the fluctuation are large (recall that we work to lowest order in the fluctuations). In the two different phases where the fluctuations are expected to be small, the two results indeed converge. As the fluctuations become smaller with increasing $N$, one expects that

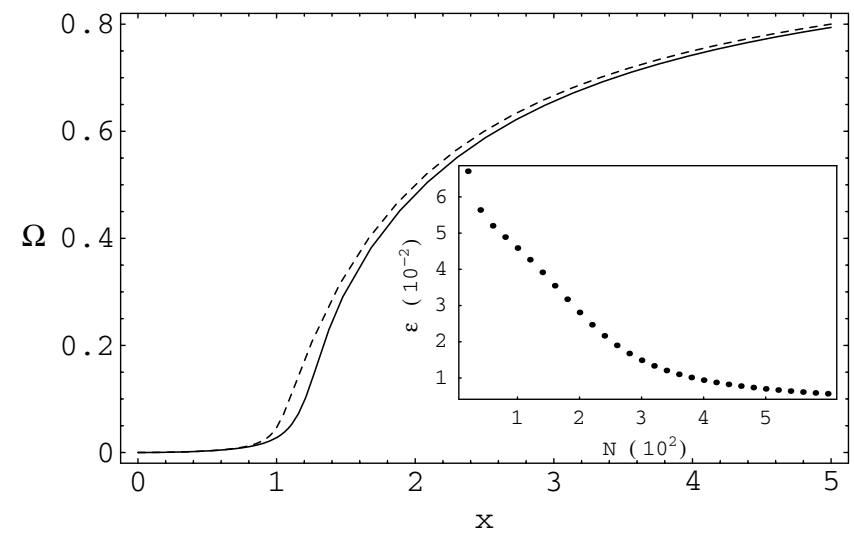

FIG. 1. Order parameter $\Omega=1+f(x) / j$ as a function of the dimensionless coupling constant $x$ for $j=N / 2=25$. The solid line is the exact result and the dashed line the result as computed from (11). The inset shows the error, $\epsilon$, as a function of $N$ for $x=1.1$. the exact and approximate results will converge upon increasing $N$. This is demonstrated in the inset of Fig. 1 where the error (absolute value of the difference between the exact and approximate results), denoted by $\epsilon$, is shown as a function of $N$ for $x=1.1$. A value of $x$ close to the transition point has been chosen as one expects the fluctuations to be the largest in this region and therefore the slowest convergence as $N$ is increased.

Once $f(x)$ has been solved for from (11), the flow equations (8) can be integrated. As mentioned before, the Hamiltonian flows to an operator diagonal in the basis of $J_{z}$. Thus we expect the off-diagonal coupling constant $\beta$ to flow to zero. Figure 2 shows the flow of $\beta$ with $j=$ 15 and for two starting values above and two below the critical point.

Since the flow is towards a diagonal form, the eigenvalues can easily be read off from the final values of $\alpha, \beta$, and $\delta$ :

$$
E_{m}=m \alpha(\infty)+j \delta(\infty), \quad m=-j,-j+1, \ldots, j .
$$

Since we linearized around the ground-state expectation value, we can at most expect this to be a good approximation to the low-lying spectrum. In particular, the ground-state energy, normalized to $j$, and gap are given by

$$
E_{g}=\delta(\infty)-\alpha(\infty), \quad \Delta=\alpha(\infty)
$$

In Fig. 3 the ground-state energy, normalized to $j$, is shown as a function of the dimensionless coupling $x$ for $j=N / 2=25$. The exact values, as obtained from a diagonalization of the Hamiltonian, are also shown. Once again very good agreement is found and, as can be expected, the results converge upon increasing $N$ since the fluctuations become weaker. This is again demonstrated in the inset which shows the error, $\epsilon$, as a function of $N$ for $x=1.1$. Note, however, that in contrast to the order parameter the agreement becomes somewhat worse at a large coupling constant, which indicates a dependence of the error on the coupling constant.

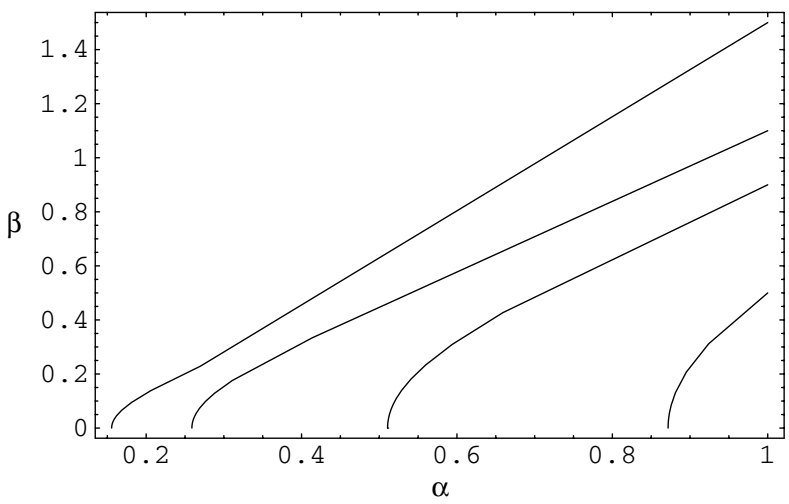

FIG. 2. Flow in the $\alpha-\beta$ plain for $j=N / 2=15$. The starting values are $\alpha=1$ in all cases and $\beta=0.5,0.9,1.1$, and 1.5, respectively. 


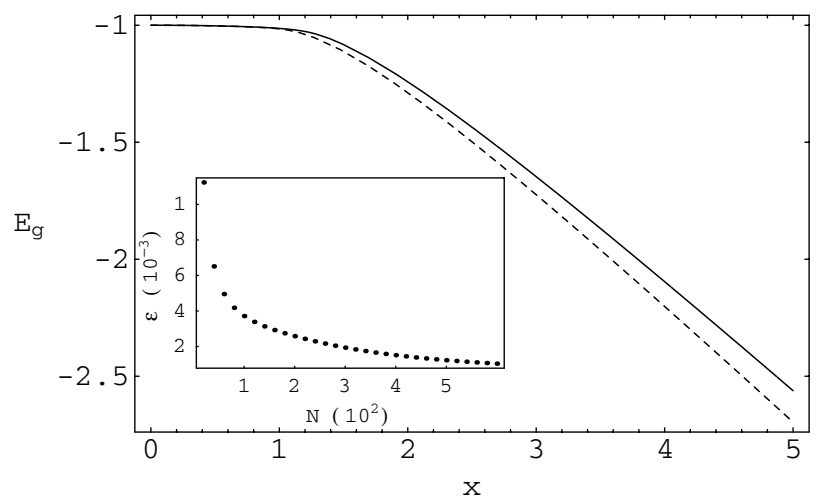

FIG. 3. Ground-state energy, normalized to $j$, as a function of the dimensionless coupling constant $x$ for $j=N / 2=25$. The solid line is the exact result and the dashed line the result as computed from (13). The inset shows the error, $\epsilon$, as a function of $N$ for $x=1.1$.

Finally, in Fig. 4 we show the gap as a function of the dimensionless coupling $x$ for $j=N / 2=25$. The exact values, as obtained from a diagonalization of the Hamiltonian, are also shown. The gap seems to be the most sensitive to the fluctuations, giving the poorest agreement of all parameters with the exact value. However, as before, increasing $N$ leads to convergence of the results due to the suppression of the fluctuations, as is illustrated in the inset which shows the error, $\epsilon$, as a function of $N$ at $x=1$.1. As with the ground-state energy the error depends on the coupling constant and becomes better at smaller coupling.

In conclusion, we have shown how the Wegner flow equations can be implemented self-consistently. In contrast with the conventional perturbative expansion in the coupling constant, this approximation explores an expansion in the fluctuations, and therefore effectively in the inverse square root of the particle number. Because of the nonanalytic behavior in the coupling constant associated with the phase transition, an expansion in the coupling constant is doomed to fail in a description of the deformed phase. On the other hand, the present approximation can avoid this difficulty to yield reasonable results in both phases for finite system sizes and small enough coupling, while convergence to the exact results is found in the thermodynamic limit. It is in this sense that the present approach is nonperturbative.

Although the order parameter at finite system size is in excellent agreement with the exact result for all parameter values, this is unfortunately not the case for the ground-state energy and gap where a dependence of the error on the coupling constant was found. This deviation cannot be explained satisfactorily in the current approximation and requires the inclusion of higher-order corrections in the fluctuations. Possible ways of dealing with this problem is to allow for a more general parametrization of the Hamiltonian [14] or by linearizing around the expectation value of $J_{z}$ in excited states in order to describe the excited spectrum, and thus the gap, more accurately [14].

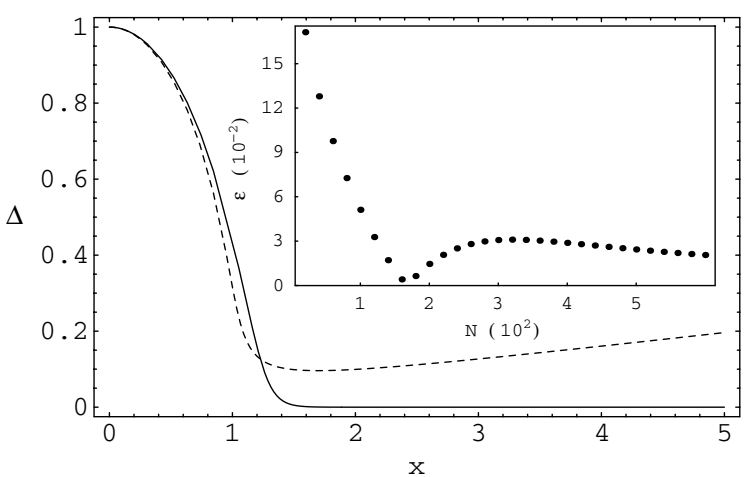

FIG. 4. Gap, $\Delta$, as a function of the dimensionless coupling constant $x$ for $j=N / 2=25$. The solid line is the exact result and the dashed line the result as computed from (13). The inset shows the error, $\epsilon$, as a function of $N$ for $x=1.1$.

As already mentioned in the introduction, the central idea, demonstrated here within the Lipkin model, can be generalized to other many-body systems, e.g., those discussed in Ref. [12] (see Ref. [14]), but the detailed implementation will depend very much on the system at hand. For this reason we have refrained from an attempt at a general presentation here, but rather focused on demonstrating the idea in a simple setting. However, one aspect generic to all systems relates to the fact that several expectation values need to be considered simultaneously in order to close the flow equations. Thus, in general, one is confronted with a coupled system of nonlinear partial differential equations. Such generalizations will be discussed elsewhere [14].

We acknowledge financial support from the South African National Research Foundation.

[1] J. Zinn-Justin, Quantum Field Theory and Critical Phenomena (Oxford University Press, Oxford, 2002).

[2] F. Wegner, Ann. Phys. 3, 77 (1994); Nucl. Phys. Proc. Suppl. B90, 141 (2000).

[3] S. D. Glazek and K. G. Wilson, Phys. Rev. D 49, 4214 (1994); 57, 3558 (1998).

[4] S. K. Kehrein, A. Mielke, and P. Neu, Z. Phys. B 99, 269 (1996).

[5] J. Stein, J. Stat. Phys. 88, 487 (1997).

[6] S. K. Kehrein, Nucl. Phys. B592, 512 (2001).

[7] A. B. Bylev and H. J. Pirner, Phys. Lett. B 428, 329 (1998)

[8] H. J. Pirner and B. Friman, Phys. Lett. B 434, 231 (1998).

[9] J. Stein, J. Phys. G 26, 377 (2000).

[10] A. Mielke, Eur. J. Phys. B 5, 605 (1998).

[11] H. J. Lipkin, N. Meshkov, and A. J. Glick, Nucl. Phys. 62, 188 (1965); 62, 199 (1965); 62, 211 (1965).

[12] T. Stauber and A. Mielke, cond-mat/0209643.

[13] P. Ring and P. Schuck, The Nuclear Many-Body Problem (Springer-Verlag, New York, 1980), p. 197.

[14] F. G. Scholtz, B. H. Bartlett, and H. B. Geyer (to be published). 